\title{
Fiber guiding at the Dirac frequency beyond photonic bandgaps
}

\author{
Kang Xie $^{1}$, Wei Zhang ${ }^{1}$, Allan D Boardman ${ }^{2}$, Haiming Jiang ${ }^{1}$, Zhijia Hu ${ }^{1,3}$, Yong Liu ${ }^{4}$, Ming Xie $^{2}$, Qiuping Mao $^{1}$, \\ Lei $\mathrm{Hu}^{1}$, Qian $\mathrm{Li}^{1}$, Tianyu Yang ${ }^{1}$, Fei Wen ${ }^{1}$ and Erlei Wang ${ }^{1}$
}

Light trapping within waveguides is a key practice of modern optics, both scientifically and technologically. Photonic crystal fibers traditionally rely on total internal reflection (index-guiding fibers) or a photonic bandgap (photonic-bandgap fibers) to achieve field confinement. Here, we report the discovery of a new light trapping within fibers by the so-called Dirac point of photonic band structures. Our analysis reveals that the Dirac point can establish suppression of radiation losses and consequently a novel guided mode for propagation in photonic crystal fibers. What is known as the Dirac point is a conical singularity of a photonic band structure where wave motion obeys the famous Dirac equation. We find the unexpected phenomenon of wave localization at this point beyond photonic bandgaps. This guiding relies on the Dirac point rather than total internal reflection or photonic bandgaps, thus providing a sort of advancement in conceptual understanding over the traditional fiber guiding. The result presented here demonstrates the discovery of a new type of photonic crystal fibers, with unique characteristics that could lead to new applications in fiber sensors and lasers. The Dirac equation is a special symbol of relativistic quantum mechanics. Because of the similarity between band structures of a solid and a photonic crystal, the discovery of the Dirac-point-induced wave trapping in photonic crystals could provide novel insights into many relativistic quantum effects of the transport phenomena of photons, phonons, and electrons.

Light: Science \& Applications (2015) 4, e304; doi:10.1038/lsa.2015.77; published online 19 June 2015

Keywords: band structure; Dirac equation; optical fiber; optical mode; photonic crystal

\section{INTRODUCTION}

Optical resonators and waveguides are widely used in modern optics ${ }^{1}$, including optical couplers/filters ${ }^{2-4}$, microlasers ${ }^{5,6}$, and mainstream optical fibers $^{7-10}$. Any dramatic change to the building blocks will generate a completely new range of optical devices. The study of the fundamental properties of resonators and waveguides thus attracts much of the current research ${ }^{1}$. Field confinement in optical fibers is traditionally based on total internal reflection. A high-index core surrounded by a cladding with a lower refractive index has been the most basic requirement ${ }^{7}$. Recently, a novel class of optical fibers that permit the guidance of light in a low-index core region has emerged ${ }^{8-10}$. These so-called photonic-bandgap fibers operate through bandgap effects of photonic crystals $^{11,12}$, which occur because of periodic microstructuring of the dielectric in the cladding region. Photonic crystals have been studied extensively for their bandgap ${ }^{1}$ and special in-band dispersion effects such as negative refraction ${ }^{13}$. However, another really interesting feature of photonic crystals is the Dirac points that appear at corners of the Brillouin zone ${ }^{14-17}$. A Dirac point, as illustrated in Figure 1, is a conical singularity surrounded by a region of linear dispersion in the band structure of triangular, hexagonal, or kagome lattices. At this special point, the Maxwell equations can be replaced by the two-dimensional massless Dirac equation for relativistic particles: $-i v_{D}\left(\sigma_{x} \partial_{x}+\sigma_{y} \partial_{y}\right) \Psi=\left(\omega-\omega_{D}\right) \Psi$, where $v_{D}$ is the group velocity,
$\omega_{D} / 2 \pi$ is the Dirac frequency, $\sigma_{x}$ and $\sigma_{y}$ are Pauli matrices, and $\Psi=\left[\begin{array}{l}\psi_{1} \\ \psi_{2}\end{array}\right]$ represents wave functions of two degenerate Bloch states.

Thus, analogies with the electronic energy band structure of graphene can be sustained ${ }^{18}$. Pseudo-diffusive transmission in such lattices has been observed ${ }^{15,16}$. A localized mode has also recently been identified at the Dirac frequency ${ }^{17}$. However, the localized mode observed thus far is non-propagating because the solution addressed in Ref. 17 is uniform in the longitudinal direction. The most common waveguides based on triangular lattices are photonic crystal fibers ${ }^{8-10}$. In the study of the fiber problem, the mode is confined in the transverse plane while carrying electromagnetic energy in the longitudinal direction; thus, the propagating effect along the fiber axis is essential for these devices to operate. The fiber problem cannot be considered two-dimensional by fixing $k_{z}$, the wave vector in the longitudinal direction, to zero. In this study, we explore the quasi-two-dimensional system. We demonstrate the discovery of a new type of light trapping by the Dirac point of photonic crystal structures for out-of-plane propagation $\left(k_{z} \neq 0\right)$. A new type of photonic crystal fibers can be created based on it. This finding provides a sort of advancement in conceptual understanding over the conventional photonic crystal fibers that rely on either total internal reflection or bandgaps. 


\section{MATERIALS AND METHODS}

Calculations of band structures by the plane wave expansion method

A typical cross-section of the photonic crystal fiber is shown in Figure 1a, where the first Brillouin zone of the corresponding reciprocal lattice is shown as an inset. The fiber is formed from either silica glass $\left(\varepsilon_{r}=1.45^{2}\right)$, germania glass $\left(\varepsilon_{r}=1.59^{2}\right)$, or SF6 glass $\left(\varepsilon_{r}=1.8^{2}\right)$. The cladding consists of a triangular lattice of air holes $\left(r_{a}=0.47 a\right)$, where $a=2.21 \mu \mathrm{m}$ is the center-to-center distance between the two nearest holes. A central hollow defect $(R=1.9 a)$ serves as the core of the fiber. The associated band structures of the triangular lattice for three different $k_{z}$ are calculated by the plane wave expansion (PWE) $\operatorname{method}^{1}$, and the results are presented in Figure 1b-1d. Variations of the Dirac point versus $k_{z}$ are plotted in Figure $2 \mathrm{a}-2 \mathrm{c}$ for the three different fibers. Guided modes of the fibers are also studied by the PWE method, where a $14 \times 16$ supercell is used for the calculation to include the fiber core as a defect of the photonic crystal. The results for the fundamental guided modes are presented in Figure $2 a-2 c$ as red diamonds. Some details of the mode profiles are given in Figure 3. As a very basic property of photonic crystal fibers, the fundamental mode consists of an approximately degenerate pair of orthogonal, quasilinearly polarized states ${ }^{9}$.

\section{Finite-difference time-domain (FDTD) numerical simulations}

In nonmagnetic media, a complete description of electromagnetic waves in time and space is given by the Maxwell equations $\nabla \times E=-\mu_{0} \frac{\partial H}{\partial t}, \nabla \times H=\varepsilon_{0} \varepsilon_{r} \frac{\partial E}{\partial t}$. The Maxwell equations can be solved directly by the well-known finite-difference time-domain
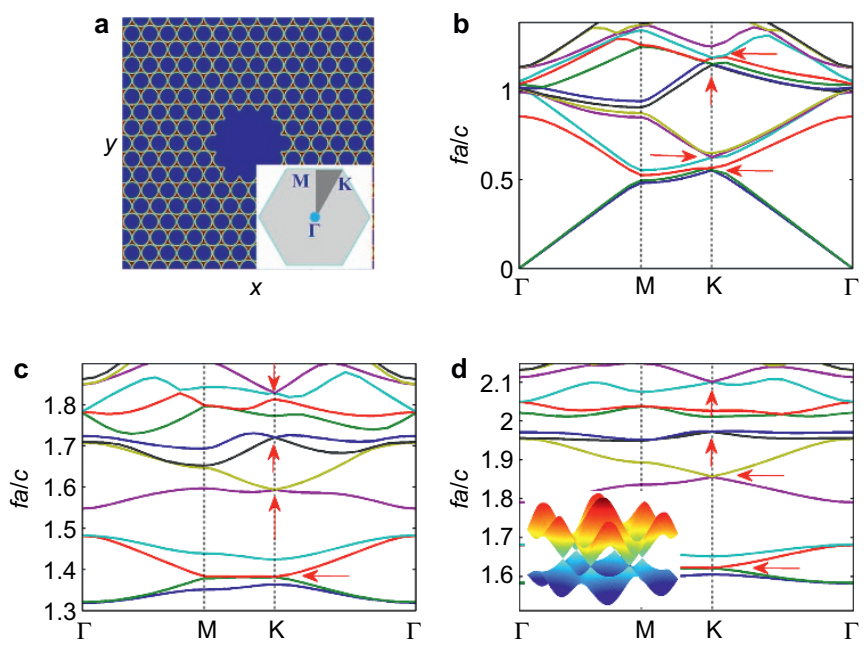

Figure 1. Cross-section and band structures of photonic crystal fiber. (a) A typical cross-section of photonic crystal fibers, where the lattice constant is $a=2.21 \mu \mathrm{m}$, the hole radius is $r_{a}=0.47 a$, and the central hollow defect radius is $R=1.9 a$. The fiber is made of either silica glass $\left(\varepsilon_{r}=1.45^{2}\right)$, germania glass $\left(\varepsilon_{r}=1.59^{2}\right)$, or SF6 glass $\left(\varepsilon_{r}=1.8^{2}\right)$. The inset in a is the first Brillouin zone (shaded light gray) of the reciprocal lattice for the triangular lattice of air holes, revealing the high-symmetry points $\Gamma, \mathrm{M}, \mathrm{K}$ at the corners of the irreducible Brillouin zone (shaded dark gray). (b-d) Band structures of the fiber cladding made of germania glass, at $k_{z} a / 2 \pi=0.001$ (b), $k_{z} a / 2 \pi=1.581$ (c), and $k_{z} a / 2 \pi=1.910$ (d). The four lowest order Dirac points are indicated by red arrows. A well-isolated, wide-open Dirac spectrum is shown in $\mathbf{d}$ in the band structure for $k_{z} a / 2 \pi=1.910$. The inset to $\mathbf{d}$ shows an enlarged three-dimensional view around the second Dirac point, where the two bands touch as a pair of linear Dirac cones at the six Brillouin zone corners.
FDTD method $^{19}$ without invoking the functional form $\exp \left(i \omega t-i k_{z} z\right)$. A three-dimensional FDTD model was established with a perfectly matched layer boundary condition on all the edges. The structure is uniform in the longitudinal direction, extending from $z=0 \mu \mathrm{m}$ to $z=1200 \mu \mathrm{m}$. On the transverse plane, the structure has a profile of the refractive index given by Figure 1a, with an area of $66.3 \mu \mathrm{m} \times 65.07 \mu \mathrm{m}$ to include 30 layers and 34 layers in the $x$ and $y$ directions, respectively. The step sizes in space are $\Delta x=\Delta z=$ $a / 20=0.1105 \mu \mathrm{m}$ and $\Delta y=a \times \sqrt{3 / 36} \approx 0.1063 \mu \mathrm{m}$. The step size in time is $\Delta t=0.1995 \mathrm{fs}$, which satisfies the Courant-Friedrichs-Levy condition $c \Delta t \leqslant\left(1 / \Delta x^{2}+1 / \Delta y^{2}+1 / \Delta z^{2}\right)^{-1 / 2}$, where $c$ is the velocity of light. For the study of transmission spectrum, a broadband Gaussian pulse is input from the front end of the fiber. The incident pulse has a Gaussian amplitude profile in the transverse plane, with a waist radius equal to the central hollow core radius of the fiber. The incident pulse has a finite duration in time, with a Gaussian spectrum centered at the Dirac frequency $\omega_{D} / 2 \pi$ and a frequency span of $0.5 \omega_{D} / 2 \pi$. This wave is simulated to evolve in the $1200-\mu \mathrm{m}$-long germania glass fiber for $8000 \mathrm{fs}$, which is long enough for the waves to pass through the structure in all directions. The full-time response at the rear end of the fiber is recorded. The transmission spectra are obtained from the timedomain response by Fourier transform. The outcomes presented in Figure $2 \mathrm{~d}-2 \mathrm{f}$ reveal peaks at frequencies inside the bandgap and at the Dirac frequency. To investigate evolutions of the different frequency components in detail, waves at specific frequencies are picked out for further simulations. For this purpose, the incident field of a continuous Gaussian beam (single harmonic) is used. The waist radius of the beam in the transverse plane is again set equal to the radius of the fiber core. The wave is simulated to evolve for long enough time to reach a steady state. The field at the end of the simulation ( $t=8000 \mathrm{fs})$ is recorded by a monitor in the $y z$ cut plane, and the results are presented in Figure 4 for four different frequency components. Leakage-free propagation of a guided mode at the Dirac frequency is visualized, confirming the transmission spectrum of Figure 2e.

\section{RESULTS AND DISCUSSIONS}

\section{Dirac cone for fiber-like propagation}

The fiber problem is different from the two-dimensional problem addressed in Ref. 17 mainly for two reasons. First, the propagation direction in the fiber is perpendicular to the lattice direction. The restriction on the longitudinal propagation wave vector $k_{z}$ must be lifted. Second, the refractive index contrast of a photonic crystal fiber is relatively small. Here, the refractive index contrast is not a free parameter to select but is dictated by the constituent materials of the fiber, which is in turn dependent on the available technology. Silica glass and germania glass are the most common materials for fiber fabrication. Other types of glasses such as SF6 can also be used to extrude a specially designed fiber ${ }^{20}$. A well-developed Dirac cone has been shown to exist in the in-plane $\left(k_{z}=0\right)$ band structure of a photonic crystal consisting of a triangular lattice of air columns in a GaAs substrate (refractive index contrast of $4-1)^{17}$. However, the Dirac cone degrades as the refractive index contrast decreases, indicating that a small refractive index contrast is a disadvantage. We begin our analysis by revealing the existence of well-developed Dirac cones in structures with refractive index contrasts as small as that of glass and air for out-of-plane propagation, which is the actual situation for fiber-like propagation. The band structure of an air-germania triangular lattice (index contrast of 1.59-1) is studied using the PWE method for various $k_{z}$. Figure $1 \mathrm{~b}$ displays the band structure of the lattice for $k_{z} a / 2 \pi=0.001$. The lowest four Dirac points are shown clustered two 

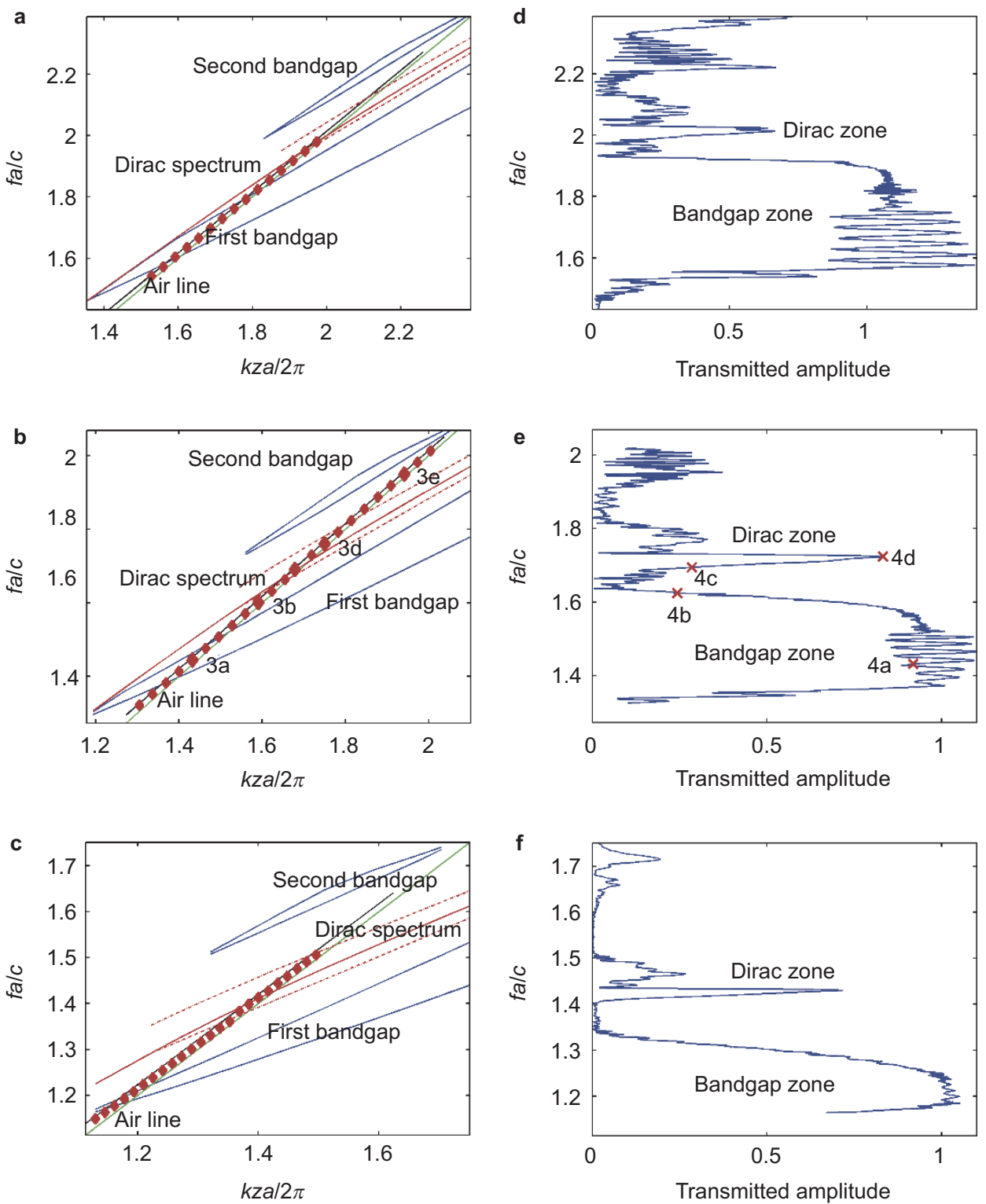

Figure 2. Propagation diagrams and transmission spectra of photonic crystal fibers. All the fibers have the same cross-section as that shown in Figure 1 a, where $a=2.21 \mu \mathrm{m}, r_{a}=0.47 a$, and $R=1.9 a$. (a-c) Propagation diagrams for the silica glass fiber $\left(\mathbf{a}, \varepsilon_{r}=1.45^{2}\right)$, germania glass fiber $\left(\mathbf{b}, \varepsilon_{r}=1.59^{2}\right)$, and $\mathrm{SF} 6$ glass fiber $\left(\mathbf{c}, \varepsilon_{r}=1.8^{2}\right)$. The solid red line represents the Dirac frequency of the fiber cladding. The dashed red lines mark the upper and lower edges of the Dirac spectrum. The black line is the theoretically calculated dispersion curve of the fundamental fiber mode obtained using the analytical model (1). The red diamonds are the numerically calculated data points of the fundamental fiber mode obtained using the PWE method. Field distributions of the modes at points $3 a-3 e$, indicated by larger diamonds in b, are presented in Figure 3a-3e. The blue curves mark the edges of the photonic bandgaps. The green line represents the light line $k_{z}=\omega / c$ of air. (d-f) Numerically simulated (by FDTD) transmission spectra of narrow pulses passing through a section (1200 $\mu \mathrm{m}$ in length) of the silica glass fiber (d), germania glass fiber (e), and SF6 glass fiber (f). Propagation scenarios of modes at the four points $4 a-4 d$, marked by red crosses in $\mathbf{e}$, are presented in Figure 4a-4d.

by two, with none of them well isolated from other bands. The situation improves as $k_{z}$ increases. The band structure for $k_{z} a / 2 \pi=1.581$ is displayed in Figure 1c. The second Dirac point, the one that involves the fifth and sixth bands, has moved away from the others. This trend continues, as is evident by the band structure shown in Figure 1d for $k_{z} a / 2 \pi=1.910$, where the second Dirac point stands out and evolves into a pair of cones. A three-dimensional plot of the second Dirac point is presented as an inset to Figure 1d, revealing the existence of well-developed Dirac cones with an outstretched Dirac spectrum for propagation out of the plane. The Dirac spectrum is defined as the frequency spectrum of linear dispersion around the Dirac point in the band structure. This example illustrates how a dwarf Dirac cone evolves into a well-developed Dirac cone as $k_{z}$ increases. This phenomenon is possible because the longitudinal wave vector $k_{z}$ strongly flattens the bands, thus leaving space for the Dirac cones. Consequently, the air-glass index contrast is sufficient to open the Dirac cone in these flatter bands with an appreciable frequency range of linear dispersion.

To better visualize the influence of $k_{z}$ on shape of the Dirac cone and to determine the threshold for the opening of the Dirac spectrum, let's concentrate on the second Dirac point and examine its process 

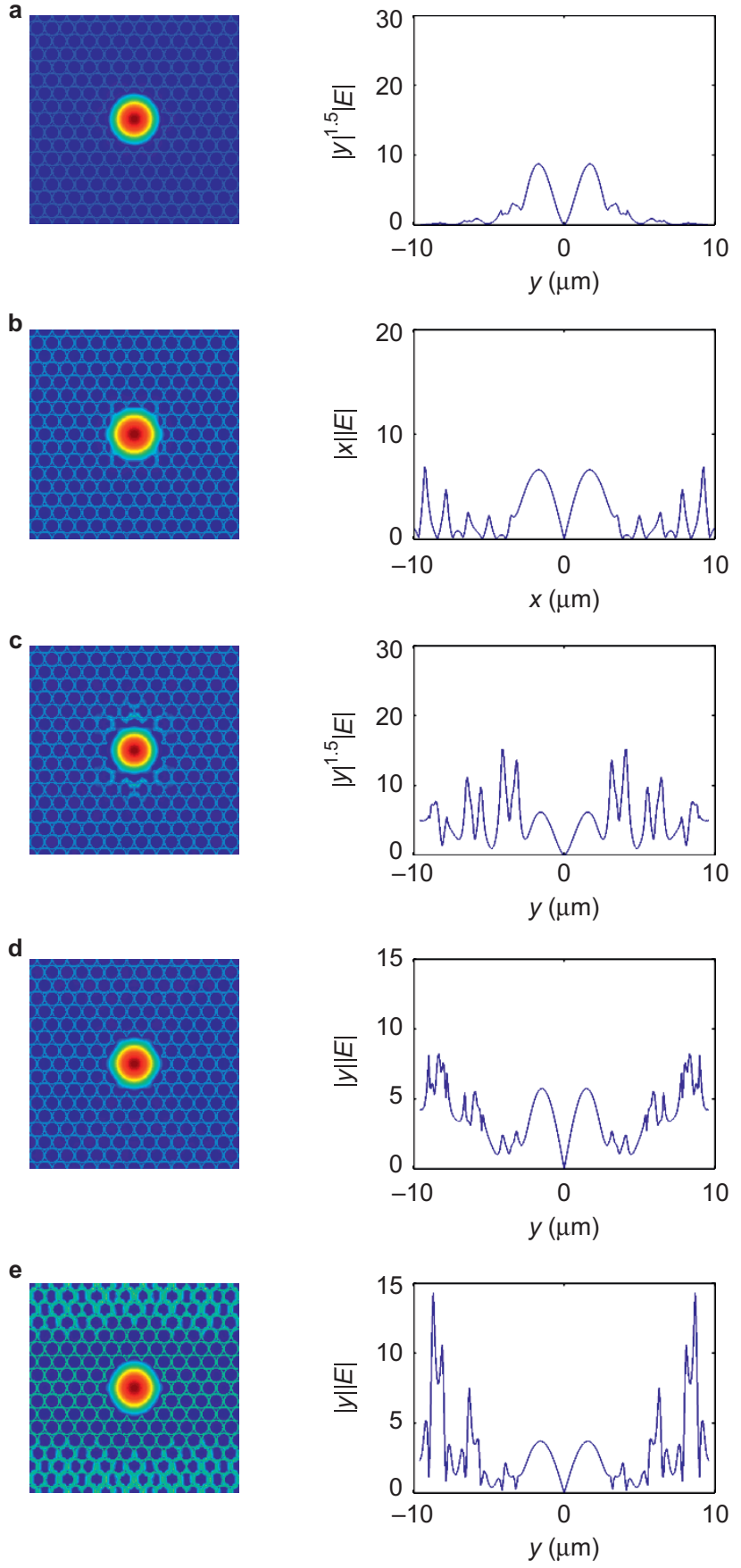

Figure 3 Guided modes in a triangular lattice photonic crystal fiber of germania glass. The fiber structure is shown in Figure 1a, where $a=2.21 \mu \mathrm{m}, r_{a}=0.47 a$, $R=1.9 a$, and $\varepsilon_{r}=1.59^{2}$. The modes were numerically calculated using the PWE method. The light-blue circles in the dark blue background give the positions of the hole-glass interfaces. (a-e) correspond to the red diamonds labeled $3 a-3 e$ in Figure $2 \mathrm{~b}$ : (a) a gap mode $(\omega \mathrm{a} / 2 \pi c=1.432)$, (b) a mode at a frequency between the photonic band gap and the lower edge of the Dirac spectrum $(\omega a / 2 \pi c=1.601)$, (c) the Dirac mode ( $\omega a / 2 \pi c=1.689$ ), (d) a mode in the Dirac spectrum ( $\omega \mathrm{a} /$ $2 \pi c=1.761$ ), and (e) a mode at a frequency higher than the upper edge of the Dirac spectrum ( $\omega a / 2 \pi c=1.950)$. The electric-field pattern $|E|$ is shown on the left, and the product $r^{3 / 2}|E|$ or $r|E|$ on the $x$-or $y$-axis is shown on the right.

of transition with the change of $k_{z}$. Starting from Figure 1d for $k_{z} a / 2 \pi=1.910$, it is apparently visible in the band structure that a linear double cone spectrum is formed by the fifth and sixth bands.
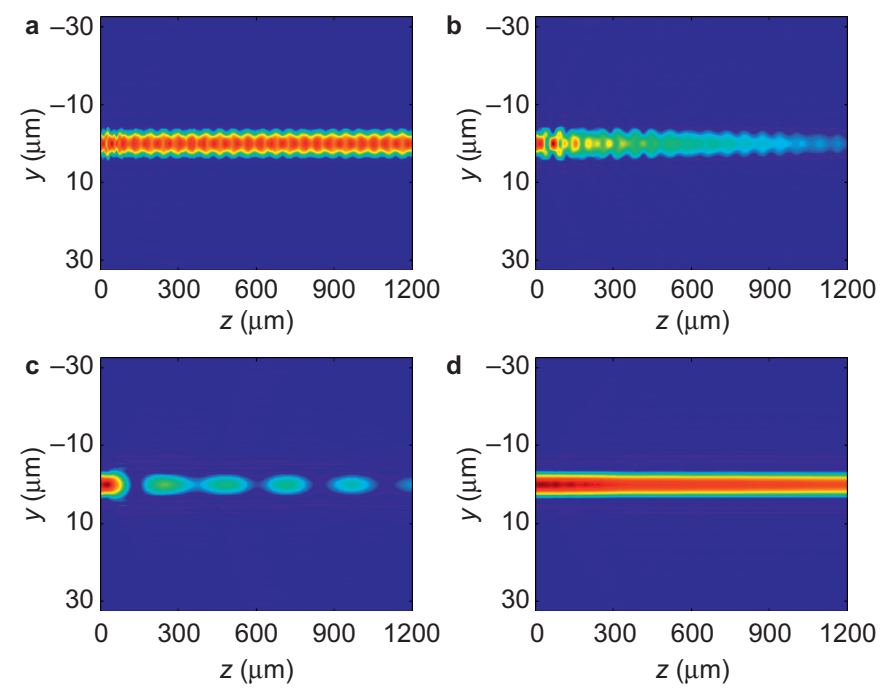

Figure 4. The steady-state electric fields in the germania fiber at the end of the FDTD simulations. The fiber has the cross-section structure shown in Figure 1a, where $a=2.21 \mu \mathrm{m}, r_{a}=0.47 a, R=1.9 a$, and $\varepsilon_{r}=1.59^{2}$. The $|E|$ fields are recorded at $t=8000 \mathrm{fs}$ and displayed by a monitor in the $y z$ cut plane that runs directly through the center axis of the fiber at $x=0$, ranging from $z=0$ to $z=1200 \mu \mathrm{m}$ in the $z$-direction and from $y=-32.535$ to $y=32.535 \mu \mathrm{m}$ in the $y$-direction. (a-d) correspond to the four red crosses labeled $4 \mathrm{a}-4 \mathrm{~d}$ in Figure 2e: (a) $|E|$ field for $\omega a / 2 \pi c=1.432$, (b) $|E|$ field for $\omega a / 2 \pi c=1.624$, (c) $|E|$ field for $\omega a / 2 \pi c=1.694$, and (d) $|E|$ field for $\omega a / 2 \pi c=1.724$.

However, as $k_{z} a / 2 \pi$ decreases, ends of the fifth band start to bend upwards in relative to the Dirac point, and thus, the frequency range of the linear spectrum around the Dirac point is reduced. When $k_{z} a / 2 \pi=1.581$, height of the magenta curve at the M point reaches that of the Dirac point, as shown in Figure 1c. In this situation, the frequency of the Dirac point overlaps with the frequency of the lower branch of the Dirac cone at the M point, eliminating the special effect of the conical singularity (vanishing density of states) altogether; thus, in effect the frequency range of the linear spectrum in the vicinity of the Dirac frequency disappears completely. As $k_{z} a / 2 \pi$ decreases further, the fifth band bends up to a higher frequency than the Dirac frequency. The Dirac frequency remains buried in frequencies of the lower branch of the Dirac cone, and the Dirac spectrum is not clearly defined, as for Figure $1 b$. The value $k_{z} a / 2 \pi=1.581$ at the turning point is taken as the threshold for the opening of the Dirac spectrum.

The above discussion suggests that well-developed Dirac cones are obtainable for photonic crystal fibers at suitable $k_{z}$. Among the four Dirac points indicated by the red arrows in Figure 1, only the second Dirac point develops a wide open Dirac spectrum. Width of the Dirac spectrum remains zero for the other three Dirac points. Since these dwarf Dirac cones cannot support localized mode, they are ignored in the subsequent discussions. The dependence of the second Dirac spectrum as $k_{z}$ varies is plotted in Figure $2 \mathrm{a}-2 \mathrm{c}$ for fibers of silica glass, germania glass, and SF6 glass, respectively. A conspicuous Dirac spectrum appears in the band structure as $k_{z}$ increases above a threshold value, where the solid red line intersects one of the dashed red lines. The threshold value of $k_{z}$ for the opening of the Dirac spectrum varies from $k_{z} a / 2 \pi \approx 1.878$ for the silica/air structure (Figure $2 \mathrm{a}$ ) to $k_{z} a / 2 \pi \approx 1.222$ for the SF6/air structure (Figure $2 \mathrm{c}$ ), depending critically on the refractive index contrast of the fiber cladding. The Dirac spectrum is also dependent on the air hole radius. For the best performance, we require $0.45 a<r_{a}<0.49 a$. 


\section{Wave localization at the Dirac frequency}

The Dirac spectrum of the fiber cladding enables a new type of guiding for waves at the Dirac frequency. Guided modes of the fibers are studied by the PWE method on a supercell. The eigen frequency of the fundamental guided mode versus $k_{z} / 2 \pi$ is plotted in Figure $2 \mathrm{a}-2 \mathrm{c}$ as red diamonds for the silica glass fiber, germania glass fiber, and SF6 glass fiber. In Figure 2d-2f, the FDTD simulated amplitude spectra of narrow pulses transmitted through a section of these fibers are plotted. For a hollow-core fiber, the mode energy is largely contained in the center air hole region; therefore, the effective index of the guided mode is always smaller than unity. For this reason, the guided modes in Figure $2 \mathrm{a}-2 \mathrm{c}$ cannot appear below the air line and instead follow the air line closely as the frequency varies. For the photonic crystal fiber made of silica glass, the refractive index contrast is so small that the opening of the Dirac spectrum occurs at large wave vector $k_{z} / 2 \pi$. It is apparent from Figure 2a that the Dirac spectrum starts so close to the air line that vast majority of the Dirac spectrum appears below the air line. The Dirac cone above the air line in this fiber is yet immature. It may not function well to sustain the Dirac mode. Indeed, the mode profile calculated at the Dirac frequency for this fiber reveals a large residual field outside the core. This opinion is supported by the results from the FDTD simulations. The FDTD simulated amplitude spectrum in Figure 2d exhibits a dwarf peak of transmission in the region of the 'Dirac zone'. Both results suggest that a photonic crystal fiber made of silica glass is not suitable for Dirac mode propagation. The Dirac spectrum opens up at smaller wave vector $k_{z} / 2 \pi$ in the photonic crystal fibers made of germania glass or SF6 glass. As shown in Figure 2b-2c, the Dirac spectrum of such a higher index contrast fiber is shifted to the left such that a substantial portion appears above the air line. Propagation of the Dirac mode is therefore well supported in both of the fibers. Comparing the SF6 glass fiber with the germania glass fiber, the curve of the Dirac frequency intersects the dispersion curve of the guided mode at a larger angle in Figure $2 c$ than in Figure 2b. This result implies that the bandwidth of the Dirac mode in the SF6 glass fiber is smaller than that in the germania glass fiber. This observation is confirmed in Figure $2 \mathrm{e}-2 \mathrm{f}$ by the narrower transmission peak in the 'Dirac zone' of the SF6 glass fiber than that for the germania glass fiber. It suggests that a broadband propagation of the Dirac mode is feasible by proper selection of the fiber material and careful design of the structure.

The mode that falls within the bandgap is a conventional gap mode. An example of the gap mode at a point marked 3a in Figure 2b (represented by a larger diamond) is plotted in Figure 3a. Outside the bandgap, the mode becomes a guided resonance that is leaky but retains its maximum within the air core for a relatively broad spectral range. Examples of the guided resonances at the points marked $3 \mathrm{~b}$ and $3 \mathrm{e}$ in Figure $2 \mathrm{~b}$ are plotted in Figure $3 \mathrm{~b}$ and $3 \mathrm{e}$, respectively. The fields of these modes are not well confined to the core and will leak away into the continuum of radiation states in the cladding and thus have finite propagation distances. At the Dirac frequency $\omega_{D} a / 2 \pi c=1.689$, marked $3 c$ in Figure $2 b$, the density of radiation states is zero ${ }^{21}$; therefore, the propagation distance of the resonance goes to infinity, and light becomes perfectly guided again in the fiber. The mode at this point, which is plotted in Figure $3 \mathrm{c}$ for its field distribution, is the new type of guided mode (Dirac mode) identified in this work. This state is no longer a leaky resonance but an eigen mode that does not decay in propagation. Compared with the gap mode in Figure 3a, one noticeable feature of the Dirac mode is the relatively large peripheral field surrounding the core. The field profile of the Dirac mode can be leveled up by multiplying it by $r^{3 / 2}$, as shown in Figure $3 \mathrm{c}$ by a slice cut on the $y$-axis. The almost constant level of oscillation in the envelope of the product as the tail extends outwards demonstrates that the envelope function of the new state decays algebraically as $1 / r^{3 / 2}$, rather than exponentially, with distance away from its center. It is demonstrated in Figure $3 \mathrm{a}$ that the field profile of a conventional gap mode cannot be leveled up by $r^{3 / 2}$. As the mode frequency deviates from the Dirac frequency, the density of radiation states increases linearly in the vicinity of the Dirac point and abruptly as the mode frequency goes beyond the Dirac spectrum. The mode becomes a guided resonance similar to that observed in the neighborhood of a bandgap. An example of the mode at the point marked $3 \mathrm{~d}$ in Figure $2 \mathrm{~b}$ is plotted in Figure 3d. All the leaky modes (Figure 3b, 3d and 3e) have tails that decay slower than $1 / r$, indicating that their total energies are not convergent.

The existence of the Dirac mode is reconfirmed by peaks in the transmission spectrum of the fiber. As shown in Figure 2e-2f, two transparent zones are apparent, as marked by 'Bandgap zone' and 'Dirac zone', respectively. The 'Bandgap zone' corresponds to the position of a bandgap, whereas the high transmission peak in the 'Dirac zone' is associated with the leakage-free propagation of the Dirac mode. By cross-checking Figure $2 \mathrm{e}-2 \mathrm{f}$ with Figure $2 \mathrm{~b}-2 \mathrm{c}$, a very good match of the two independent set of calculations is obtained. In Figure 2e-2f, the sharp peaks appear at $\omega a / 2 \pi c=1.724$ and 1.429 in FDTD simulations for the germania glass fiber and the SF6 glass fiber, respectively. In Figure $2 b-2 c$, which emerge from the PWE calculations, however, the intersection point of the curves of the Dirac frequency and the dispersion is at $\omega a$ l $2 \pi c=1.689$ for the germania glass fiber and at 1.415 for the SF6 glass fiber. The locations of the intersection points in Figure $2 b-2 c$ for the onset of the Dirac modes agree well with the positions of the sharp transmission peaks in Figure 2e-2f for the leakage-free propagation, leaving discrepancies of approximately $2 \%$ and $1 \%$, respectively. At a frequency near the intersection point, all the bandgaps in Figure $2 b-2 c$ are far away from the air line; thus, they are not reachable by a coreguided mode. The Dirac mode is therefore the only localized mode that can be confined to the air core in this frequency range. This situation guarantees pure Dirac-mode operation of the fiber in the 'Dirac zone', where the fiber selectively supports only the Dirac mode for leakage-free propagation.

\section{Physical mechanism of the light guiding at the Dirac frequency}

To mathematically show that an optical bound state is feasible at the Dirac frequency, we examine the governing equation of the waves in such a photonic crystal. Close to the Dirac frequency, photon transport in the transverse plane of the lattice is describable by the massless Dirac equation ${ }^{14,15}:-i v_{D}\left(\sigma_{x} \partial_{x}+\sigma_{y} \partial_{y}\right) \Psi=\left(\omega-\omega_{D}\right) \Psi$. It is well known that the Dirac equation substantiates a half-bound solution of the form $\psi_{1,2} \propto e^{ \pm i \theta} / r$ at the Dirac frequency. This type of mode has been previously studied for charge carriers in graphene ${ }^{18}$, where the wave function is the probability of finding the spinors in space. This relativistic quantum mechanics model can be adopted to optics by a slight modification to the interpretation of the modal function. In photonic crystals, the wave function $\psi_{1,2} \propto e^{ \pm i \theta} / r$ represents the envelope amplitude of a basis function (Bloch state) ${ }^{14}$, and the basis wave function intrinsic to a cylindrical geometry is a cylindrical wave function ${ }^{17} \phi \propto$ $J_{0}(K r)$, where $K=4 \pi /(3 a)$. Therefore, at the Dirac frequency, the physical quantities $E_{z}$ and $H_{z}$ in the photonic crystal are characterized by $J_{0}(K r) e^{ \pm i \theta} / r$. Because the amplitude of the Bessel function decays as $1 / \sqrt{ } r$, the overall spatial dependence of the corresponding optical state asymptotically approaches $r^{-3 / 2}$ at large distances. This result 
coincides precisely with the asymptotic algebraic spatial profile exhibited by Figure $3 c$, thus verifying the mathematical model of the Dirac equation. The $r^{-3 / 2}$ spatial profile ensures square integrability of the physical quantities and confirms that the Dirac mode in photonic crystal fibers is a well-behaved, true bound state.

As outlined in Supplementary Information, using $J_{0}(K r) e^{ \pm i \theta} / r$ for the wave solution of Maxwell equations in the photonic crystal cladding, the eigenvalue equation of the fundamental guided mode of the fiber is derived as

$$
\begin{gathered}
\left(W_{1}\left(k_{1} R\right)-W_{0}(K R)+\frac{1}{K^{2} R^{2}}\right)\left(W_{1}\left(k_{1} R\right)-\varepsilon_{\text {eff }} W_{0}(K R)+\varepsilon_{\text {eff }} \frac{1}{K^{2} R^{2}}\right) \\
=\frac{k_{z}^{2}}{k_{0}^{2}}\left(\frac{1}{k_{1}^{2} R^{2}}-\frac{1}{K^{2} R^{2}}\right)^{2},
\end{gathered}
$$

where $k_{0}=\omega / c, k_{1}^{2}=k_{0}{ }^{2}-k_{z}^{2}, \varepsilon_{e f f}=\left(K^{2}+k_{z}^{2}\right) / k_{0}^{2}, \quad W_{N}(x)=\frac{J_{N}{ }^{\prime}(x)}{x J_{N}(x)}$.

Upon solving Equation (1) for the eigen frequency $k_{0} c / 2 \pi$ as a function of the wave vector $k_{z} / 2 \pi$, the dispersion relation of the guided mode is obtained. The results are plotted in Figure $2 a-2 c$ as black lines for the three different fibers. Good agreement with numerical results is observed. The analytical model (1) is derived at the Dirac frequency $\omega_{D} / 2 \pi$. When applied to a frequency $\omega / 2 \pi$ other than $\omega_{D} / 2 \pi$, we quantitatively require $\delta \omega\left(\equiv \omega-\omega_{D}\right)<<\omega_{D}$ for the result to be valid. In practice, we typically find it to be accurate for frequency within the Dirac spectrum, and often qualitatively extensible for even larger frequency range, as shown in Figure 2a-2c. For the case of germania glass fiber (Figure 2b), the black line intersects the red line at $\omega a / 2 \pi c=1.668$. This theoretical result compares well with the value $\omega a / 2 \pi c=1.689$ from the PWE calculation for the onset of the Dirac mode, leaving only a discrepancy of approximately $1.2 \%$. The electric field pattern of the mode at the Dirac frequency obtained using this analytical model has a single lobe monopole pattern inside the hollow core and a power-law profile in the surrounding cladding, which compares well with the numerically calculated field distribution presented in Figure 3c. These basic similarities between the theoretical and numerical patterns further validate the theory and numerical findings.

The trapping of light at the Dirac frequency is more intuitively recognized by examining the density of states of the waves propagating in the surrounding medium. Right at the Dirac frequency, the density of radiation states in a triangular lattice photonic crystal is precisely zero $^{21}$; thus, outgoing waves are forbidden in the surrounding medium, and field confinement to the center hollow core becomes possible. Because the zero density of states at the Dirac frequency is a special feature of the band structure, and the band structure itself is a consequence of Bragg scattering of light by the photonic crystal lattice, at a deeper level of understanding, the localization phenomenon observed at the Dirac frequency is essentially due to Bragg scattering, which determines the overall band structure. Rather than leading to a complete gap, however, we find an isolated conical singularity where the band gap is vanishingly small. The key feature of this band structure is that the two bands touch as a pair of cones at corners of the Brillouin zone in a linear fashion, with linear dispersion and a linearly vanishing density of states in its vicinity. One would expect to see a true localized mode precisely at the point where the density of states is zero and a guided resonance with finite lifetime away from it. The fact that there is not a band of true localized modes around the Dirac frequency gives the mode special properties such as an algebraic profile and sharp frequency response. This light-trapping phenomenon occurs at a single frequency and should be distinguished from a recent observation of an 'embedded eigenvalue ${ }^{22,23}$ that also occurs at a single frequency. The existence of the Dirac mode is due to disappearance of radiation states at the Dirac frequency, whereas the trapped light in Refs. 22 and 23 occurs in the presence of radiation states; however, somehow, mutual coupling of the trapped light and radiation states is suppressed by destructive interference. In light of this discussion, the two phenomena are different in how they break up coupling of the trapped light and radiation states.

Numerical confirmation of the guided mode at the Dirac frequency To confirm the existence of a trapped state at the Dirac frequency, numerical tools ${ }^{24}$ based on the FDTD method ${ }^{19}$ were adopted to simulate the evolutions of waves passing through a section $(1200 \mu \mathrm{m}$ long) of the germania glass fiber. In Figure $4 \mathrm{a}$, a typical gap mode propagating over this distance is presented. The oscillation in amplitude is due to a mismatch between the input wave and the eigenmode of the fiber. When the frequency goes beyond the bandgap, the mode still concentrates much of its field energy in the core. Nevertheless, the mode is no longer a true-bound state but a guided resonance that resembles the so-called ARROW mode ${ }^{25,26}$. Like the ARROW mode of a cylindrical waveguide, this mode has a $|E| \propto 1 / \sqrt{ } r$ asymptotic profile in space. It is a radiative mode itself $\left(\int|E|^{2} r d r d \theta \rightarrow \infty\right)$ and is therefore inherently leaky. An example of the guided resonance is shown in Figure $4 \mathrm{~b}$. Further away from the bandgap, the resonance is less concentrated within the center core until the resonance broadens away and melts into the continuum. As the localized mode penetrates into the region of the Dirac spectrum, the density of radiation states starts to decrease because of the presence of the Dirac point. Guided resonance similar to that observed in the neighborhood of a bandgap makes an appearance in the Dirac spectrum. The mode, such as that shown in Figure 4c, for example, remains leaky because it is situated inside a band of a continuum of states and mutual coupling is inevitable. When the frequency reaches the Dirac frequency, the density of radiation states vanishes; thus, coupling of the Dirac mode and the radiation states disappears completely. The profile of the mode thus approaches $|E| \propto r^{-3 / 2}$, which is square integrable, indicating that the mode becomes trapped again. Mode propagation at the Dirac frequency is shown in Figure 4d, an almost leakage-free propagation is achieved. The leakage-free operational window of the fiber can be best visualized by a transmission spectrum of the fiber itself, as shown in Figure 2d-2f, where the Dirac frequency corresponds to a sharp transmission peak in each case. The simulations presented in Figure $4 \mathrm{a}-4 \mathrm{~d}$ correspond to the four points $4 \mathrm{a}-4 \mathrm{~d}$ marked by red crosses in Figure 2e. From the observation of the low and high transmissivity and the corresponding locations on the transmission spectrum, Figure $4 \mathrm{a}-4 \mathrm{~d}$ is consistent with behaviors expected at the points $4 \mathrm{a}-4 \mathrm{~d}$; therefore, the propagation scenarios of these modes are understood. This finding represents an important discovery, based on which a new type of fiber guiding is established at the Dirac frequency beyond a bandgap.

If the lattice extends to infinity and material absorption is ignored, the Dirac mode in Figure $4 \mathrm{~d}$ and the gap mode in Figure $4 \mathrm{a}$ are both lossless. In practice, however, a real lattice has a finite size, which results in a loss that is associated with field penetration across the boundary into the surrounding medium. Because the tail of the gap mode decays exponentially, whereas the tail of the Dirac mode decays algebraically with distance, for the same lattice extent, the residual field of the Dirac mode on the boundary is stronger than that of the gap mode. Consequently, the Dirac mode is more lossy than a gap mode in general. Because of the leakage to the surrounding medium, propaga- 
tion at a transmission peak is not truly leakage-free in a fiber of finite cross section, but, as shown in Figure $4 \mathrm{a}$ and $4 \mathrm{~d}$, declines slowly with distance following an exponential law $P=P_{0} e^{-\alpha z}$ for its power. For the germania glass fiber, the attenuation constant $\alpha$ is estimated to be $1.5 \times 10^{-4} \mu \mathrm{m}^{-1}$ for the Dirac mode and $\sim 0$ for the gap mode. Fortunately, the loss due to the finite lattice size can be made as small as desired by increasing the number of surrounding crystal periods.

\section{Practical fiber design, experimental validation, and applications}

The design concern for the new proposed fiber is quite similar to the design concern of an ordinary photonic crystal fiber. The complexity of the photonic crystal structure denies an analytical solution; thus, a lot of computer software (see Ref. 24 for some examples) has been developed to facilitate the design of practical photonic crystal fibers. This commercially available software can be directly applied to the design activity of the new proposed fiber because it still belongs to the group of photonic crystal fibers. The only difference is a shift of attention from the bandgap to the Dirac point. To be specific, the first concern of the design is whether a Dirac mode can be supported by a fiber with given parameters. The band structure of a photonic crystal fiber can be calculated using one of the available commercial software products or using a simple MATLAB code based on the PWE method ${ }^{1}$. Our studies demonstrate that the Dirac mode is not well supported in a photonic crystal fiber if the refractive index contrast is as small as that of a silica/air $(1.45-1)$ system. We confirm that photonic crystal fibers made of SF6 glass $\left(n_{r}=1.8\right)$ or germania glass $\left(n_{r}=1.59\right)$ are suitable for the Dirac mode propagations. From these studies, the constituent materials of the fiber can be selected, and the air hole radius can be optimized. The second concern for the design is the precise position of the Dirac frequency. As shown in Figure $2 b-2 c$, the curves of the Dirac frequency and the dispersion intersect at $\omega a / 2 \pi c \approx 1.689$ for the germania glass fiber and at 1.415 for the SF6 glass fiber. The frequency at the intersection point can be used as a rough estimate of the operational frequency for leakage-free propagation of the Dirac mode. For the lattice constant $a=2.21 \mu \mathrm{m}$, these frequencies correspond to wavelengths of approximately 1308 and $1562 \mathrm{~nm}$, respectively. The selected fiber materials, germania and SF6, are transparent at these wavelengths. Note that the Dirac frequency can be scaled to any desired value by adjusting the lattice period $a$.

Experimental validation of the Dirac mode can be established by the identification of the Dirac transmission peak on spectra measured on the specially designed photonic crystal fibers. From numerical simulations the Dirac peak shows up nicely in transmission spectra of photonic crystal fibers made of SF6 glass or germania glass. The above two fibers are therefore potential media for Dirac peak transmission measurements. In addition, polymer fiber is easy to handle and widely available. The refractive index of polystyrene, which is 1.590 , is very close to that of germania glass, so polystyrene fiber is a convenient alternative for the Dirac transmission peak measurement. Experimental verification of the theory presented here is handled smoothly, and this will be the subject of a later paper.

Photonic crystal fibers were traditionally divided into two broad classes, namely, index-guiding fibers and photonic-bandgap fibers, depending on whether they used index guiding or photonic bandgaps for optical confinement. Our finding of Dirac point guiding enriches the varieties of photonic crystal fibers. Bandgap confinement is attractive because it allows light to be guided within a hollow core. This capability not only minimizes the effects of material absorptions, undesired nonlinearities, and any other unwanted properties of the bulk materials but also facilitates applications in biological sensors or organic light-emitting devices, where enhancement of laser interactions with dilute or gaseous media is required. Our new proposed fiber also allows light to be guided within a hollow core; thus, any benefit a photonic-bandgap fiber can offer is also available in the Dirac fiber. If photonic-bandgap fibers already exist; what is the purpose of exploring a new type of guiding phenomenon? The first reason is simple curiosity: the idea of using a Dirac point to trap light in a hollow core is intriguing. Second, the Dirac-point-induced guiding does have unique features. The Dirac mode is different from the gap mode in terms of the resulting frequency characteristics and spatial profile features. Our new proposed fiber can therefore be regarded as a supplement to existing photonic-bandgap fibers. For example, the unique power-law profile of the Dirac mode results in loose lateral confinement, thus enabling long-range interaction between multiple cores. This feature could lead to the creation of new coupling and sensing characteristics for use in optical couplers, laser arrays, and fiber sensors. The sharp frequency response of the Dirac mode can take on the role of a resonant cavity with the establishment of frequency selectivity in applications of coherent random fiber lasers, where resonant cavities are not available but where frequency selectivity is desired ${ }^{27}$.

\section{CONCLUSIONS}

A new type of light trapping was demonstrated in fibers at the Dirac frequency in the absence of a bandgap and total internal reflection. This discovery will extend the possibilities of fiber optics and will lead to many new applications in fiber lasing/sensing systems. We believe that the effects discovered in the present study for electromagnetic waves in photonic crystals could occur in other wave phenomenon systems, including acoustic waves in phononic crystals and matter waves in semiconductors. A further search for different systems with the extraordinary localization of photons, as well as phonons and electrons, can expand the applicability of our results.

\section{ACKNOWLEDGEMENTS}

KX and ZH acknowledge financial support by the NSFC (60588502 and 11404087). ADB acknowledges financial support by the EPSRC of UK.

1 Skorobogatiy M, Yang J. Fundamentals of Photonic Crystal Guiding. Cambridge: Cambridge University Press, 2009

2 Liu H, Zhu S. Coupled magnetic resonator optical waveguides. Laser \& Photon Rev 2013; 7: 882-900.

3 Meade RD, Devenyi A, Joannopoulos JD, Alerhand OL, Smith DA et al. Nove applications of photonic band gap materials: low-loss bends and high $Q$ cavities. J Appl Phys 1994; 75: 4753-4755.

4 Fan S, Villeneuve PR, Joannopoulos JD, Haus HA. Channel drop tunneling through localized states. Phys Rev Lett 1998; 80: 960-963.

5 Shakoor A, Savio RL, Cardile P, Portalupi SL, Gerace D et al. Room temperature allsilicon photonic crystal nanocavity light emitting diode at sub-bandgap wavelengths. Laser \& Photon Rev 2013; 7: 114-121.

6 Diao Z, Bonzon C, Scalari G, Beck M, Faist J et al. Continuous-wave vertically emitting photonic crystal terahertz laser. Laser \& Photon Rev 2013; 7: L45-L50.

7 Kao KC, Hockham GA. Dielectric-fibre surface waveguides for optical frequencies. IEEE Proc J Optoele 1986; 133: 191-198.

8 Knight JC, Broeng J, Birks TA, Russell PS. Photonic band gap guidance in optical fibers. Science 1998; 282: 1476-1478.

9 Steel MJ, White TP, de Sterke CM, McPhedran RC, Botten LC. Symmetry and degeneracy in microstructured optical fibers. Opt Lett 2001; 26: 488-490.

10 Knight JC. Photonic crystal fibres. Nature 2003; 424: 847-851.

11 Yablonovitch E. Inhibited spontaneous emission in solid-state physics and electronics. Phys Rev Lett 1987; 58: 2059-2062.

12 John S. Strong localization of photons in certain disordered dielectric superlattices. Phys Rev Lett 1987; 58: 2486-2489.

13 Gajić R, Meisels R, Kuchar F, Hingerl K. All-angle left-handed negative refraction in Kagomé and honeycomb lattice photonic crystals. Phys Rev B 2006; 73: 165310. 
14 Raghu S, Haldane FD. Analogs of quantum-Hall-effect edge states in photonic crystals. Phys Rev A 2008; 78: 033834.

15 Sepkhanov RA, Bazaliy YB, Beenakker CW. Extremal transmission at the Dirac point of a photonic band structure. Phys Rev A 2007; 75: 063813.

16 Zandbergen SR, de Dood MJ. Experimental observation of strong edge effects on the pseudodiffusive transport of light in photonic graphene. Phys Rev Lett 2010; 104: 043903.

17 Xie K, Jiang HM, Boardman AD, Liu Y, Wu ZH et al. Trapped photons at a Dirac point: a new horizon for photonic crystals. Laser \& Photon Rev 2014; 8: 583-589.

18 Castro Neto AH, Guinea F, Peres NM, Novoselov KS, Geim AK. The electronic properties of graphene. Rev Mod Phys 2009; 81: 109-162.

19 Taflove A, Hagness SC. Computational Electrodynamics: The Finite-difference Timedomain Method. Boston: Artech House, 2005.

20 Ravi Kanth Kumar VV, George AK, Reeves WH, Knight JC, Russell PS et al. Extruded soft glass photonic crystal fiber for ultrabroad supercontinuum generation. Opt Express 2002; 10: 1520-1525.

21 Plihal M, Maradudin AA. Photonic band structure of two-dimensional systems: the triangular lattice. Phys Rev B 1991; 44: 8565-8571.

22 Hsu CW, Zhen B, Lee J, Chua SL, Johnson SG et al. Observation of trapped light within the radiation continuum. Nature 2013; 499: 188-191.
23 Hsu CW, Zhen B, Chua SL, Johnson SG, Joannopoulos JD et al. Bloch surface eigenstates within the radiation continuum. Light Sci \& App 2013; 2: e84; doi:10.1038/Isa2013.40.

24 The open source MIT photonic bands software package at http://ab-initio.mit.edu/wiki/ index.php/Main_Page ; Lumerical FDTD Solutions at https://www.lumerical.com/; OptiFDTD at http://optiwave.com/; The RSoft Photonics Suite from RSoft Design Group at http://optics.synopsys.com/rsoft/.

25 Rowland KJ, Afshar S, Monro TM. Bandgaps and antiresonances in integratedARROWs and Bragg fibers; a simple model. Opt Exp 2008; 16: 17935-17951.

26 Langbein U, Trutschel U, Unger A, Duguay M. Rigorous mode solver for multilayer cylindrical waveguide structures using constraints optimization. Opt Quant Electron 2009; 41: 223-233.

27 Hu ZJ, Zhang Q, Miao B, Fu Q, Zou G et al. Coherent Random fiber laser based on nanoparticles scattering in the extremely weakly scattering regime. Phys Rev Let 2012; 109: 253901.

\section{(c) (1)}

This license allows readers to copy, distribute and transmit the Contribution as long as it attributed back to the author. Readers are permitted to alter, transform or build upon the Contribution, and use the article for commercial purposes. Please read the full license for further details at - http://creativecommons.org/licenses/by/4.0/ 